\title{
Distinct malignant behaviors of mouse myogenic tumors induced by different oncogenetic lesions
}

\author{
Simone Hettmer ${ }^{1,2,3,4}$ *, Roderick T. Bronson ${ }^{5}$ and Amy J. Wagers ${ }^{2,3,4}$ \\ 1 Division of Pediatric Hematology and Oncology, Department of Pediatric and Adolescent Medicine, University Medical Center Freiburg, Freiburg, Germany \\ ${ }^{2}$ Department of Stem Cell and Regenerative Biology, Harvard Stem Cell Institute, Harvard University, Boston, MA, USA \\ ${ }^{3}$ Howard Hughes Medical Institute, Chevy Chase, MD, USA \\ 4 Joslin Diabetes Center, Boston, MA, USA \\ ${ }^{5}$ Department of Biomedical Sciences, Tufts University Veterinary School, North Grafton, MA, USA
}

Edited by:

Thomas Grunewald, Ludwig

Maximilian University of Munich,

Germany

Reviewed by:

Frederic Barr, National Cancer

Institute, USA

Rossella Rota, Ospedale Pediatrico

Bambino Gesù, Italy

\section{*Correspondence:}

Simone Hettmer, University Medical

Center, Mathildenstrasse 1, 79106

Freiburg, Germany

e-mail:simone.hettmer@uniklinik-

freiburg.de

\begin{abstract}
Rhabdomyosarcomas (RMS) are heterogeneous cancers with myogenic differentiation features. The cytogenetic and mutational aberrations in RMS are diverse. This study examined differences in the malignant behavior of two genetically distinct and disease-relevant mouse myogenic tumor models. Kras; p1619null myogenic tumors, initiated by expression of oncogenic Kras in p16p19null mouse satellite cells, were metastatic to the lungs of the majority of tumor-bearing animals and repopulated tumors in seven of nine secondary recipients. In contrast, SmoM2 tumors, initiated by ubiquitous expression of a mutant Smoothened allele, did not metastasize and repopulated tumors in 2 of 18 recipients only. In summary, genetically distinct myogenic tumors in mice exhibit marked differences in malignant behavior.
\end{abstract}

Keywords: rhabdomyosarcoma, myogenic differentiation, metastasis, transplantation

\section{INTRODUCTION}

Rhabdomyosarcomas (RMS) are heterogeneous cancers with myogenic differentiation (1). Fusion-positive RMS tumors carry exclusive chromosomal translocations at $\mathrm{t}(2 ; 13)(\mathrm{q} 35 ; \mathrm{q} 14)$ or $\mathrm{t}(1 ; 13)(\mathrm{p} 36 ; \mathrm{q} 14)$ and exhibit aggressive clinical behavior $(2,3)$. The remaining, fusion-negative spectrum of human RMS comprises a diverse group of tumors with frequent RAS pathway activation $(4,5)$ and variable mutations, including loss of heterozygosity at the $P T C H 1$ locus $(6,7)$ in a subset of fusion-negative RMS. PTCH1 serves as a Hedgehog (Hh) receptor, and loss of PTCH1 function results in de-repression of downstream Hh pathway signaling. The contributions of RMS-relevant oncogenic pathways, including RAS and Hh signaling, to myogenic tumor formation were previously tested in mice $(8,9)$. This report highlights the distinct phenotypes of two mouse myogenic tumor models those initiated by combined Cdkn2a (p16p19) disruption and Kras expression in transplanted mouse muscle satellite cells (10) and those arising in the skeletal muscle of mice with activated Hh signaling due to expression of a mutant, constitutively active smoothened (SmoM2) allele $(11,12)$. We demonstrate significant differences in tumor-repopulating activity and prevalence of lung metastases between Kras-driven and Hh-driven myogenic tumors in mice. These observations reveal marked differences in malignant behavior between genetically distinct mouse myogenic tumors, suggesting that an understanding of the distinct oncogenetic underpinnings of tumors on the fusion-negative RMS spectrum may be informative for clinical prognosis and treatment.

\section{MATERIALS AND METHODS \\ MICE}

R26-SmoM2 (mixed genetic background including 129/Sv and Swiss Webster as main components) (11), CAGGS-CreER (11), and NOD.CB17-Prkdc $c^{\text {scid } / J ~(N O D . S C I D) ~ m i c e ~ w e r e ~ p u r c h a s e d ~}$ from The Jackson Laboratory. p16p19 ${ }^{\text {null }}$ mice (B6.129 background) were obtained from the NIH/Mouse Models of Human Cancer Consortium. Mice were bred and maintained at the Joslin Diabetes Center Animal Facility. All animal experiments were approved by the Joslin Diabetes Center Institutional Animal Care and Use Committee.

\section{SARCOMA INDUCTION}

Kras; $p 16 p 19^{\text {null }}$ myogenic tumors were initiated by fluorescenceactivated cell sorting of $p 16 p 19^{\text {null }}$ satellite cells, followed by lentiviral transduction to introduce oncogenic $\operatorname{Kras}(G 12 v)$ and implantation in the gastrocnemius muscles of NOD.SCID mice as previously described (10). R26-SmoM2;CAGGS-CreER were injected with Tamoxifen $(1 \mathrm{mg} / 40 \mathrm{~g})$ on postnatal day 10 to activate expression of CRE recombinase and SMOM2. R26-SmoM2;CAGGS-CreER spontaneously developed multifocal skeletal muscle tumors (SmoM2 tumors) as previously described $(11,12)$.

\section{HISTOPATHOLOGY}

Tumor tissue was dissected, fixed in $4 \%$ paraformaldehyde for $2 \mathrm{~h}$, and embedded in paraffin. Standard H\&E stained sections were prepared. Staining for Actin (Dako, M0635, 1:200), Desmin (Dako, M0760, 1:50), and Ki67 Ki67 (Vector Labs, VP-K451, 1:250) was performed as previously described (10).

\section{LUNG METASTASES}

Tumor-bearing mice were monitored at least twice weekly for health problems, and were sacrificed once tumors reached a volume of $1 \mathrm{~cm}^{3}$ or were ill. Lungs were dissected, fixed in $4 \%$ paraformaldehyde for $2 \mathrm{~h}$, and embedded in paraffin. Standard 
$H \& E$ stained sections were prepared and evaluated for the presence of metastases by Roderick T. Bronson.

\section{TUMOR TRANSPLANTATION}

Tumors were harvested, digested in DMEM $+0.2 \%$ collagenase type II (Invitrogen) $+0.05 \%$ dispase (Invitrogen) for $90 \mathrm{~min}$ at $37^{\circ} \mathrm{C}$ in a shaking waterbath, triturated to disrupt the remaining tumor pieces, and filtered through a $70 \mathrm{~mm}$ cell strainer. Red blood cells were lysed from tumor cell preparations by $3 \mathrm{~min}$ incubation in $0.15 \mathrm{M}$ ammonium chloride, $0.01 \mathrm{M}$ potassium bicarbonate solution on ice. Defined numbers of tumor cells were resuspended in $10-15 \mathrm{ml}$ of HBSS with $2 \%$ FBS and injected into the gastrocnemius muscles of 1 - to 3-month-old, anesthetized NOD.SCID mice using a transdermally inserted dental needle attached to a Hamilton syringe via polyethylene tubing. Recipient muscles were preinjured $24 \mathrm{~h}$ before cell implantation by injection of $25 \mathrm{ml}$ of a $0.03 \mathrm{mg} / \mathrm{ml}$ solution of cardiotoxin (from Naja mossambica, Sigma) in order to enhance cell engraftment. Mice were screened once weekly for the development of tumors at the injection sites.

\section{STATISTICS}

Differences between Kras; p16p19 $9^{\text {null }}$ and SmoM2 mouse myogenic tumors were evaluated by T-test (Ki67 indices), Fisher's Exact test (prevalence of lung metastases), and Kaplan-Meier analysis (tumor-repopulating activity).

\section{RESULTS}

\section{Kras; p16p19null AND SmoM2 MOUSE TUMORS EXHIBIT A MYOGENIC TUMOR PHENOTYPE}

Kras; $p 16 p 19^{\text {null }}$ mouse myogenic tumors were induced by intramuscular implantation of $\operatorname{Kras}(G 12 v)$-expressing $p 16 p 19^{\text {null }}$ muscle satellite cells (10). In contrast, SmoM2 mouse myogenic tumors were initiated by ubiquitous activation of a mutant, constitutively active smoothened (SmoM2) allele in R26-SmoM2;CAGGS-CreER mice $(11,12)$. The phenotypes of Kras; $p 16 p 19^{n u l l}$ and SmoM2 myogenic tumors were previously described (10-12). In brief, Kras; p16p19null tumors contained bundles of cells with large, atypical nuclei, frequent mitotic figures, and occasional multinucleated giant cells. Subsets of cells ( $<50 \%$ of all tumor cells) expressed terminal muscle differentiation markers such as desmin and actin (Figure 1A), and the proliferative index as evidenced by the percentage of Ki67-expressing nuclei was $41.6 \pm 12.5 \%$ (range 30.5-59.3\%; four tumors evaluated) (Table 1). SmoM2 tumors contained many multinucleated, elongated cells with abundant cytoplasm interspersed with small round cells. SmoM2 tumors lacked cellular atypia and diffusely expressed desmin and actin in many tumor cells (more than $75 \%$ of all tumor cells; Figure 1B). As previously reported (12), the Ki67 index of SmoM2 tumors was $19.1 \pm 15.9 \%$ (range 3.4-41.8\%; six tumors evaluated) and lower than that observed in Kras; $p 16 p 19^{\text {null }}$ tumors $(p=0.05$; Table 1).

\section{Kras; p16p19null AND SmoM2 MOUSE MYOGENIC TUMORS HAVE DIFFERENT METASTATIC POTENTIAL}

The lung is the primary organ affected by distant sarcoma metastases in humans. To assess the metastatic potential of Kras; p16p19null and SmoM2 tumors, random lung sections obtained

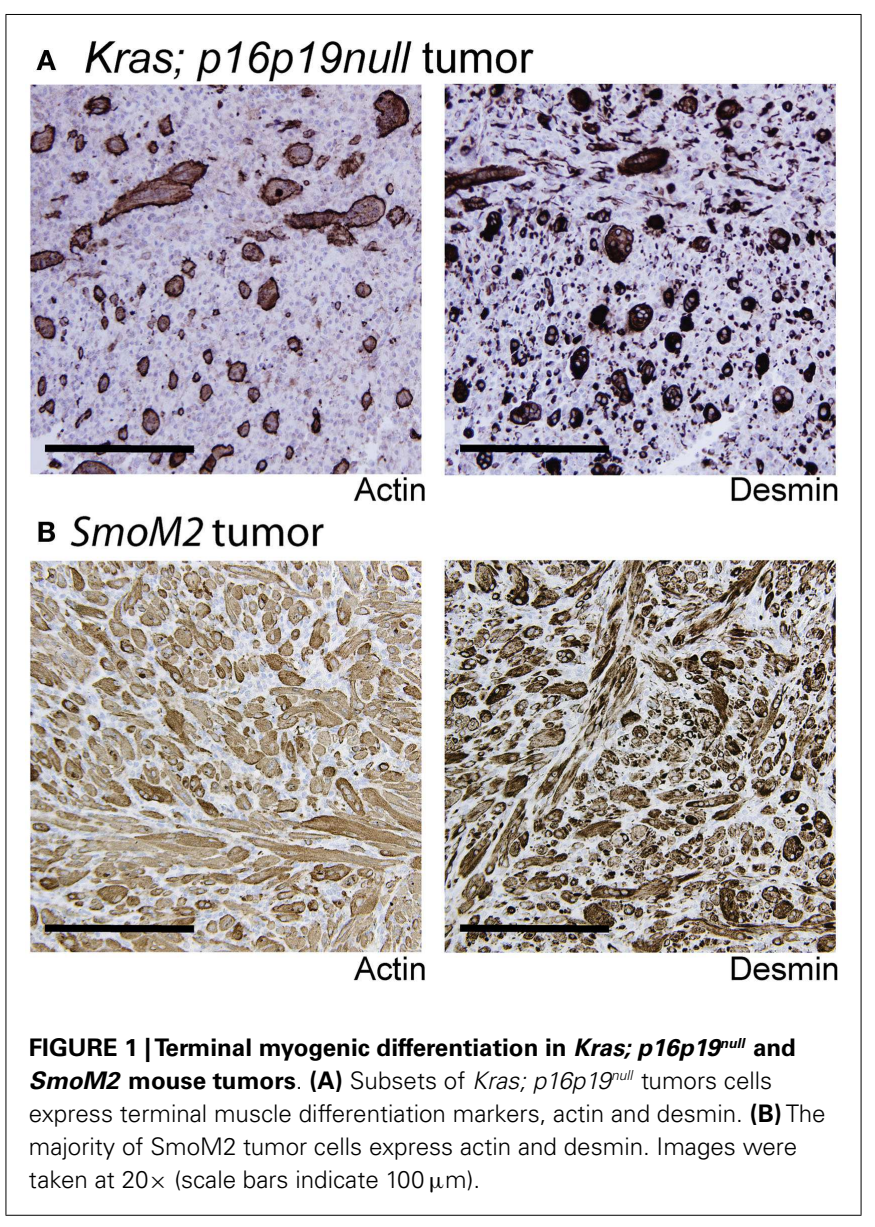

from tumor-bearing animals were screened for the presence of metastases. Six of seven mice with Kras; p16p19 $19^{\text {null }}$ myogenic tumors were found to have lung metastases at the time of death (mice were sacrificed 17-28 days after detection of palpable tumors) (Figure 2). In contrast, 0 of 8 mice with SmoM2 myogenic tumors had lung metastases at the time of death (mice were sacrificed at 38-55 days of age and 5-21 days after detection of palpable tumors). The prevalence of lung metastases in Kras; $16 p 19^{\text {null }}$ and SmoM2 myogenic tumor-bearing mice was significantly different $(p=0.001)$.

\section{Kras; p16p19null AND SmoM2 MOUSE MYOGENIC TUMORS DIFFER IN TUMOR-REPOPULATING ACTIVITY}

Most malignant tumors contain cells that have the capacity to repopulate secondary tumors when transplanted into a susceptible secondary environment, and this assay has been used as a test of the malignancy of distinct tumors and tumor cell subsets (13). To evaluate the tumor-repopulating activity of Kras; $16 p 19^{\text {null }}$ and SmoM2 mouse myogenic tumors, viable tumor cells were transplanted into the cardiotoxin-pre-injured gastrocnemius muscles of NOD.SCID mice. The Kras; $p 16 p 19^{n u l l}$ tumor cell pool contains approximately $70 \% \mathrm{GFP}+$ cells and $30 \% \mathrm{GFP}$ - cells (10). Because tumor-repopulating activity in Kras; $p 16 p 19^{\text {null }}$ tumors resides within the Kras-expressing, GFP + subset of tumor cells descended from virally infected satellite cells (Figure S1 in Supplementary 
Table 1 | Differences in the malignant behavior of Kras; p16p19null and SmoM2 mouse tumors.

\begin{tabular}{|c|c|c|}
\hline & Kras; p16p19null tumors & SmoM2 tumors \\
\hline Terminal muscle differentiation & Actin/desmin expression in $<50 \%$ of tumor cells & Actin/desmin expression in $>75 \%$ of tumor cells \\
\hline Ki67 index $(p=0.05)$ & $41.6 \pm 12.5 \%$ & $19.1 \pm 15.9 \%$ \\
\hline Metastases $(p=0.001)$ & 7 of 9 mice with lung metastases & 0 of 10 mice with lung metastases \\
\hline Transplantation $(p<0.001)$ & 7 of 9 transplanted mice developed tumors (50 cells injected) & $\begin{array}{l}2 \text { of } 10 \text { transplanted mice developed secondary } \\
\text { tumors (100-150 k cells injected) }\end{array}$ \\
\hline
\end{tabular}

Kras; p16p19null and SmoM2 mouse myogenic tumors exhibit profound differences in tumor-repopulating activity and metastatic behavior.

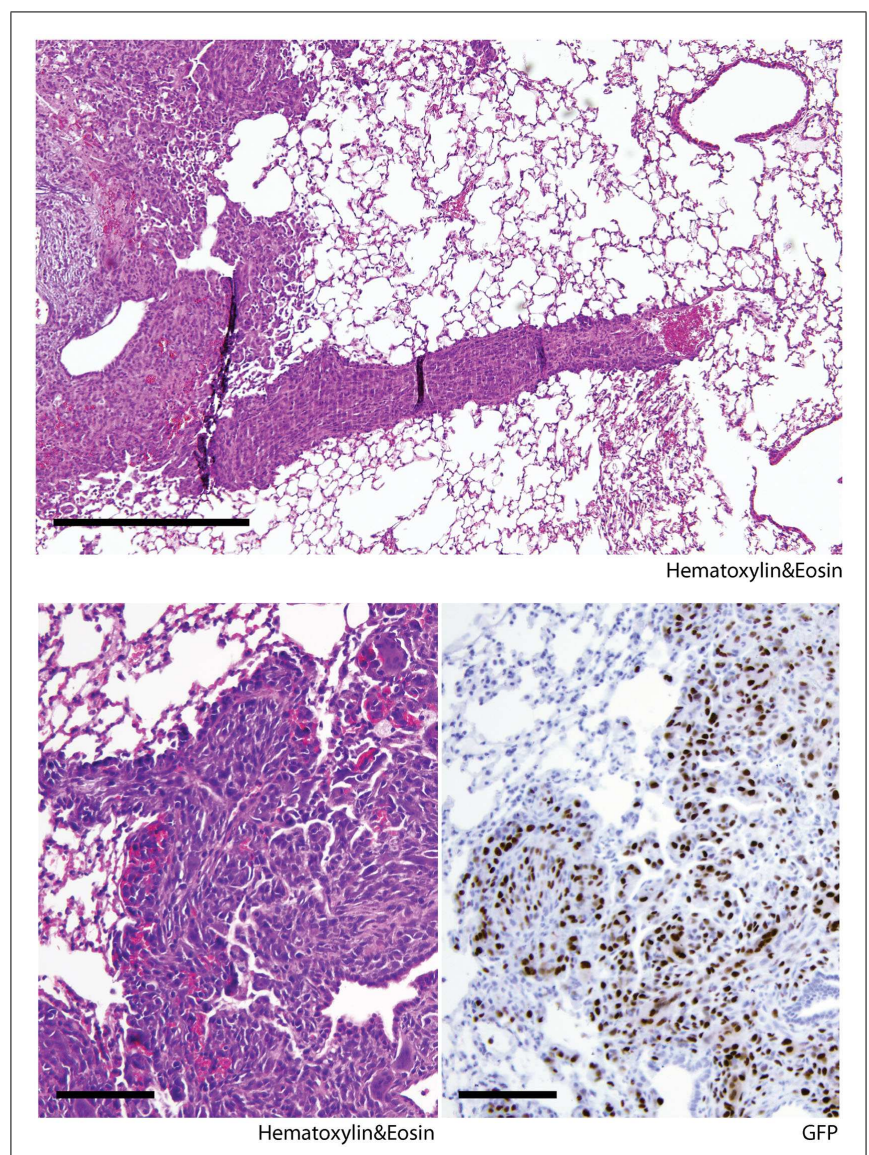

FIGURE 2 | Kras; p16p19null mouse myogenic tumors metastasize to the lungs of tumor-bearing animals. Random lung sections from Kras; p16p19null tumor-bearing mice show metastases. Tumor cells invade lung capillaries (top panel). Similar to primary tumors arising from GFP+ Kras-expressing; $p 16 p 19^{\text {null }}$ satellite cells, lung metastases are GFP+ (bottom right panel). Images were taken at $10 \times$ and $20 \times$ (scale bars indicate $100 \mu \mathrm{m}$ )

Material), Kras; p16p19 $9^{\text {null }}$ tumor cells were sorted for transplantation from two Kras; $16 p 19^{\text {null }}$ primary tumors as GFP+, Pi-, Calcein + cells. Seven of nine mice injected with only $50 \mathrm{GFP}+$, $\mathrm{Pi}-$, Calcein + Kras; p16p19 $9^{\text {null }}$ tumor cells developed secondary tumors at the injection site 26-39 days after tumor cell injection. For SmoM2 tumors, viable tumor cells were sorted as PICalcein + cells from primary tumors obtained from four mice. Surprisingly, despite significantly higher numbers of cells transplanted
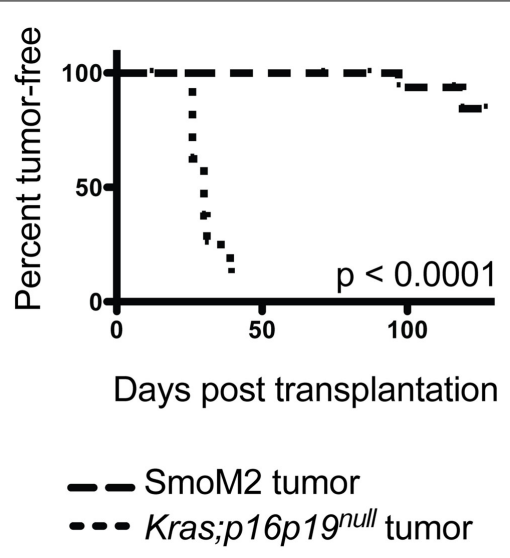

FIGURE 3 | Kras; p16p19null tumor cells repopulate tumors in secondary recipients more effectively than SmoM2 mouse tumor cells.

$\mathrm{Pi}^{-} \mathrm{Ca}^{+} \mathrm{GFP}^{+}$Kras; $p 16 p 19^{\text {null }}$ tumor cells were sorted independently from two primary tumors and injected into the cardiotoxin-pre-injured gastrocnemius muscles of NOD.SCID mice (50 cells per injection). Pi-Ca+ SmoM2 tumor cells were sorted independently from four primary tumors and injected into the cardiotoxin-pre-injured gastrocnemius muscles of NOD.SCID mice (100,000-150,000 cells per injection). Recipient mice were monitored for the occurrence of secondary tumors at the injection site for up to 4 months.

$(100,000$ to $150,000 \mathrm{PI}-$, Calcein + SmoM2 tumor cells per recipient), only 2 of 18 recipient mice developed secondary tumors, which were detected 71 and 127 days after cell injection. These experiments indicate marked differences in tumor-repopulating activity of Kras; $p 16 p 19^{\text {null }}$ and SmoM2 tumors $(p<0.001$, Figure 3), in terms of both the frequency of tumor-repopulating cells and the latency of secondary tumor formation.

\section{DISCUSSION}

Our findings highlight differences in the malignant phenotype and behavior of mouse myogenic tumors driven by activation of distinct RMS-relevant oncogenic pathways. Kras; $p 1619^{\text {null }}$ myogenic tumors were metastatic to the lungs of the majority of tumorbearing animals and contained high tumor-repopulating activity. In contrast, SmoM2 tumors did not metastasize and were substantially less effective in repopulating tumors in secondary recipients. These observations indicate that genetically distinct myogenic tumors in mice display marked differences in their malignant behavior. 
The two model systems described in this study were induced by different experimental methods. SmoM2 tumors originated from Cre-mediated activation of a conditionally expressed transgene. Kras; p16p19 $19^{\text {null }}$ mouse tumors, on the other hand, were initiated by viral transduction and intramuscular implantation of target satellite cells. We note that Kras; Tp53-/- mouse myogenic tumors $(14,15)$, induced by Cre-mediated activation of oncogenic hits instead of viral transduction, exhibit a phenotype that closely resembles the Kras; $p 16 p 19^{\text {null }}$ mouse tumors described here. For example, Kras; $p 16 p 19^{\text {null }}$ share their propensity to metastasize to the lungs of tumor-bearing animals with Kras; $T p 53^{-/-}$mouse tumors (14). Nevertheless, it is possible that differences in the tumor induction strategy (such as off-target effects of viral transduction) could contribute to the observed differences in malignant behavior between SmoM2 and Kras; p16p19 $19^{\text {null }}$ mouse myogenic tumors.

Similar to mouse myogenic tumors, human fusion-negative RMS comprises a group of tumors with clear differences in histology, myogenic differentiation state, oncogenic pathway activation, and genetic background. In recent years, subsets of human RMS tumors that exhibit a combination of specific genetic and phenotypic characteristics were distinguished. For example, a subset of human fusion-negative RMS with spindle cell/sclerosing histology was recently found to exhibit diffuse MyoD expression, carry frequent somatic MyoD mutations, and portend a poor prognosis (16, 17). Also, children with TP53 germline mutations are predisposed to develop anaplastic RMS at a young age (18), and germline mutations in DICER1 were linked to a genetic susceptibility to develop RMS of the genitourinary tract (19). Future extended (epi-)genotype/phenotype correlations might pinpoint clinically/biologically distinct subgroups of human fusion-negative RMS and identify biomarkers to facilitate prognostication and/or stratification of therapy.

\section{AUTHOR CONTRIBUTIONS}

$\mathrm{SH}, \mathrm{RB}$, and $\mathrm{AW}$ conceived experiments, analyzed data, wrote, and approved of the manuscript.

\section{ACKNOWLEDGMENTS}

We thank C. L. Unitt and T. Bowman at the DF/HCC Histopathology Core for help with immunohistochemistry, D. Tchessalova for excellent animal care, and Joyce LaVecchio, Girijesh Burizula, and Atsuya Wakayabashe in the Joslin Diabetes Center Flow Cytometry Core (supported by the Harvard Stem Cell Institute and NIH P30DK036836) for flow cytometry support. This work was funded in part by a Stand Up To Cancer-American Association for Cancer Research Innovative Research Grant (SU2C-AACR-IRG1111; to AW); by grants from the Burroughs-Wellcome Fund and the Harvard Stem Cell Institute (to AW), and by P.A.L.S. Bermuda/St. Baldrick's, ALSF, and Bear Necessities (to SH). Content is solely the responsibility of the authors and does not necessarily represent the official views of the NIH or other funding agencies.

\section{SUPPLEMENTARY MATERIAL}

The Supplementary Material for this article can be found online at http://www.frontiersin.org/Journal/10.3389/fonc.2015. 00050/abstract

\section{REFERENCES}

1. Parham DM. Pathologic classification of rhabdomyosarcomas and correlations with molecular studies. Mod Pathol (2001) 14:506-14. doi:10.1038/modpathol. 3880339

2. Kumar S, Perlman E, Harris CA, Raffeld M, Tsokos M. Myogenin is a specific marker for rhabdomyosarcoma: an immunohistochemical study in paraffinembedded tissues. Mod Pathol (2000) 13:988-93. doi:10.1038/modpathol. 3880179

3. Sorensen PH, Lynch JC, Qualman SJ, Tirabosco R, Lim JF, Maurer HM, et al. PAX3-FKHR and PAX7-FKHR gene fusions are prognostic indicators in alveolar rhabdomyosarcoma: a report from the children's oncology group. J Clin Oncol (2002) 20:2672-9. doi:10.1200/JCO.2002.03.137

4. Chen X, Stewart E, Shelat AA, Qu C, Bahrami A, Hatley M, et al. Targeting oxidative stress in embryonal rhabdomyosarcoma. Cancer Cell (2013) 24:710-24. doi:10.1016/j.ccr.2013.11.002

5. Shern JF, Chen L, Chmielecki J, Wei JS, Patidar R, Rosenberg M, et al. Comprehensive genomic analysis of rhabdomyosarcoma reveals a landscape of alterations affecting a common genetic axis in fusion-positive and fusion-negative tumors. Cancer Discov (2014) 4:216-31. doi:10.1158/2159-8290.CD-13-0639

6. Bridge JA, Liu J, Weibolt V, Baker KS, Perry D, Kruger R, et al. Novel genomic imbalances in embryonal rhabdomyosarcoma revealed by comparative genomic hybridization and fluorescence in situ hybridization: an intergroup rhabdomyosarcoma study. Genes Chromosomes Cancer (2000) 27:337-44. doi:10.1002/(SICI) 1098-2264(200004)27:4<337::AID-GCC1>3.0.CO;2-1

7. Tostar U, Malm CJ, Meis-Kindblom JM, Kindblom LG, Toftgard R, Unden AB. Deregulation of the hedgehog signalling pathway: a possible role for the PTCH and SUFU genes in human rhabdomyoma and rhabdomyosarcoma development. J Pathol (2006) 208:17-25. doi:10.1002/path.1882

8. Rubin BP, Nishijo K, Chen HI, Yi X, Schuetze DP, Pal R, et al. Evidence for an unanticipated relationship between undifferentiated pleomorphic sarcoma and embryonal rhabdomyosarcoma. Cancer Cell (2011) 19:177-91. doi:10.1016/j. ccr.2010.12.023

9. O'Brien D, Jacob AG, Qualman SJ, Chandler DS. Advances in pediatric rhabdomyosarcoma characterization and disease model development. Histol Histopathol (2012) 27:13-22.

10. Hettmer S, Liu J, Miller CM, Lindsay MC, Sparks CA, Guertin DA, et al. Sarcomas induced in discrete subsets of prospectively isolated skeletal muscle cells. Proc Natl Acad Sci U S A (2011) 108:20002-7. doi:10.1073/pnas.1111733108

11. Mao J, Ligon KL, Rakhlin EY, Thayer SP, Bronson RT, Rowitch D, et al. A novel somatic mouse model to survey tumorigenic potential applied to the Hedgehog pathway. Cancer Res (2006) 66:10171-8. doi:10.1158/0008-5472. CAN-06-0657

12. Hettmer S, Teot LA, Van Hummelen P, Macconaill L, Bronson RT, Dall'osso C, et al. Mutations in Hedgehog pathway genes in fetal rhabdomyomas. J Pathol (2013) 231(1):44-52. doi:10.1002/path.4229

13. Lapouge G, Beck B, Nassar D, Dubois C, Dekoninck S, Blanpain C. Skin squamous cell carcinoma propagating cells increase with tumour progression and invasiveness. EMBO J (2012) 31:4563-75. doi:10.1038/emboj.2012.312

14. Kirsch DG, Dinulescu DM, Miller JB, Grimm J, Santiago PM, Young NP, et al. A spatially and temporally restricted mouse model of soft tissue sarcoma. Nat Med (2007) 13:992-7. doi:10.1038/nm1602

15. Blum JM, Ano L, Li Z, Van Mater D, Bennett BD, Sachdeva M, et al. Distinct and overlapping sarcoma subtypes initiated from muscle stem and progenitor cells. Cell Rep (2013) 5:933-40. doi:10.1016/j.celrep.2013.10.020

16. Kohsaka S, Shukla N, Ameur N, Ito T, Ng CK, Wang L, et al. A recurrent neomorphic mutation in MYOD1 defines a clinically aggressive subset of embryonal rhabdomyosarcoma associated with PI3K-AKT pathway mutations. Nat Genet (2014) 46:595-600. doi:10.1038/ng.2969

17. Yasui N, Yoshida A, Kawamoto H, Yonemori K, Hosono A, Kawai A. Clinicopathologic analysis of spindle cell/sclerosing rhabdomyosarcoma. Pediatr Blood Cancer (2014). doi:10.1002/pbc.25367

18. Hettmer S, Archer NM, Somers GR, Novokmet A, Wagers AJ, Diller L, et al. Anaplastic rhabdomyosarcoma in TP53 germline mutation carriers. Cancer (2014) 120:1068-75. doi:10.1002/cncr.28507

19. Doros L, Yang J, Dehner L, Rossi CT, Skiver K, Jarzembowski JA, et al. DICER1 mutations in embryonal rhabdomyosarcomas from children with and without familial PPB-tumor predisposition syndrome. Pediatr Blood Cancer (2012) 59:558-60. doi:10.1002/pbc. 24020 
Conflict of Interest Statement: The authors declare that the research was conducted in the absence of any commercial or financial relationships that could be construed as a potential conflict of interest.

Received: 21 December 2014; accepted: 11 February 2015; published online: 24 February 2015.

Citation: Hettmer S, Bronson RT and Wagers AJ (2015) Distinct malignant behaviors of mouse myogenic tumors induced by different oncogenetic lesions. Front. Oncol. 5:50. doi: $10.3389 /$ fonc. 2015.00050
This article was submitted to Pediatric Oncology, a section of the journal Frontiers in Oncology.

Copyright (c) 2015 Hettmer, Bronson and Wagers. This is an open-access article distributed under the terms of the Creative Commons Attribution License (CC $B Y)$. The use, distribution or reproduction in other forums is permitted, provided the original author(s) or licensor are credited and that the original publication in this journal is cited, in accordance with accepted academic practice. No use, distribution or reproduction is permitted which does not comply with these terms. 